\title{
Neurofibromas plexiformes voluminosos de cuello en la neurofibromatosis tipo 1
}

\author{
Ignacio Pascual-Castroviejo, Samuel I. Pascual-Pascual, Ramón Velázquez-Fragua, Juan Viaño, \\ Diana Quiñones-Tapia, Fernando López-Barea
}

Objetivo. Presentar las características clínicas, de imagen y evolutivas de una serie de pacientes con neurofibromatosis tipo 1 que desarrollaron durante la infancia neurofibromas plexiformes voluminosos en el cuello (NFPVC).

Pacientes y métodos. Nueve pacientes (cinco mujeres y cuatro varones) con edades entre 3 y 15 años en el momento del diagnóstico de los tumores, que podían extenderse también a la fosa posterior y a la zona torácica superior. El diagnóstico estuvo basado fundamentalmente en la clínica, la imagen y la histología.

Resultados. Un tumor era intralaríngeo y causaba problemas respiratorios. Los otros ocho casos tenían su origen en varias raíces espinales de uno o de ambos lados y podían crecer también hacia el interior de la fosa posterior y de la región torácica en algunos pacientes, y desplazaban a las estructuras anatómicas vecinas, especialmente en tres casos, todos niñas, en las que el tumor creció hasta alcanzar gran volumen, especialmente por un lado, parándose el crecimiento entre los 11 y 12 años y no volviendo a crecer más tarde.

Conclusiones. Los NFPVC son tumores histológicamente benignos. La extirpación es necesaria cuando están localizados en la laringe por los problemas respiratorios que causan, pero no en los de las otras regiones, pese a que el voluminoso tamaño que alcanzan en algunos casos puede causar exagerados desplazamientos de las estructuras vecinas. El estudio de nuestra serie parece indicar que al menos los tumores extralaríngeos sólo crecen hasta los 11-12 años. Puede ser recomendable retrasar la cirugía tanto como sea posible si no existe sintomatología aguda que la haga necesaria.

Palabras clave. Neurofibroma plexiforme. Neurofibroma plexiforme de laringe. Neurofibroma plexiforme de mediastino. Neurofibroma plexiforme voluminoso del cuello. Neurofibromatosis 1. Trastornos neurocutáneos.

\section{Introducción}

La neurofibromatosis tipo 1 (NF1) (OMIM 62299) es un trastorno neurocutáneo, de herencia autosómica dominante, caracterizado por una amplia variabilidad fenotípica. El diagnóstico de NF1 se realiza de acuerdo con la conferencia NIHCD de 1988 [1]. La NF1 tiene una incidencia de 1 entre 3.000 a 4.000 individuos y la causan alteraciones del gen NF1 localizado en el cromosoma 17q11.2 [2]. Los neurofibromas plexiformes (NFP) son tumores de la vaina de los nervios periféricos que se originan de los nervios subcutáneos, espinales y viscerales periféricos o autonómicos, y contienen todos los elementos de los nervios periféricos. Los NFP se presentan desde el nacimiento [3] o se desarrollan a lo largo de los primeros años de la vida. Histológicamente, los NFP se caracterizan por una proliferación de las células de Schwann en la vaina nerviosa a lo largo del nervio, y afectan a múltiples fascículos y múltiples nervios, creciendo en la matriz endoneural. Aunque el NFP se puede encontrar en cualquier parte del cuerpo, tumores del cuello, inclu- yendo faringe, laringe, cavidad oral o incluso en la región intratorácica, se publican raramente, como se ponía en evidencia en una relativamente reciente revisión de la bibliografía [4]. Series con tres [5] y cuatro [6,7] pacientes pediátricos son las más numerosas aportadas hasta la fecha. La mayoría de los NFP del cuello, faringe, laringe y áreas torácicas más altas se describen en niños y adultos jóvenes [8-12].

Dado que estos tumores son de crecimiento lento, generalmente poco dolorosos, y tienen pocos o ningún síntoma clínico, su reconocimiento se puede retrasar algunos años, hasta que aparecen las manifestaciones clínicas de la afectación faríngea o laríngea. En nuestra serie, sólo un tumor localizado en la laringe causó sintomatología clínica, dificultades respiratorias, para ponernos sobre el camino del diagnóstico. En el resto de los pacientes, el tumor fue un hallazgo radiológico tras el estudio practicado por la NF1.

Los NFP pueden alcanzar un gran tamaño a lo largo de los primeros años, antes de la adolescencia, a los que vamos a considerar como NFP voluminosos de cuello (NFPVC), sin atrevernos a eti-
Servicio de Neurología Pediátrica (I. Pascual-Castroviejo, S.I. PascualPascual, R. Velázquez-Fragua); Departamento de Anatomía Patológica (F. López-Barea); Hospital Universitario La Paz. Unidad de Imagen; Hospital del Rosario (J. Viaño, D. Quiñones-Tapia) Madrid, España.

Correspondencia:

Dr. Ignacio Pascual Castroviejo. Orense, 14, 10. E. E-28020 Madrid.

E-mail:

pascualcastroviejo@gmail.com

Aceptado tras revisión externa: 14.01.14.

Cómo citar este artículo: Pascual-Castroviejo I, PascualPascual SI, Velázquez-Fragua R, Viaño J, Quiñones-Tapia D, López-Barea F. Neurofibromas plexiformes voluminosos de cuello en la neurofibromatosis tipo 1. Rev Neurol 2014; 59: 13-9.

(c) 2014 Revista de Neurología 
Figura 1. Caso 3: tumor de laringe en un niño de 12 años. El plano del estudio de resonancia magnética enriquecido con contraste muestra un neurofibroma plexiforme de laringe bilateral externo y unilateral interno en el lado izquierdo (flecha) del cuello.

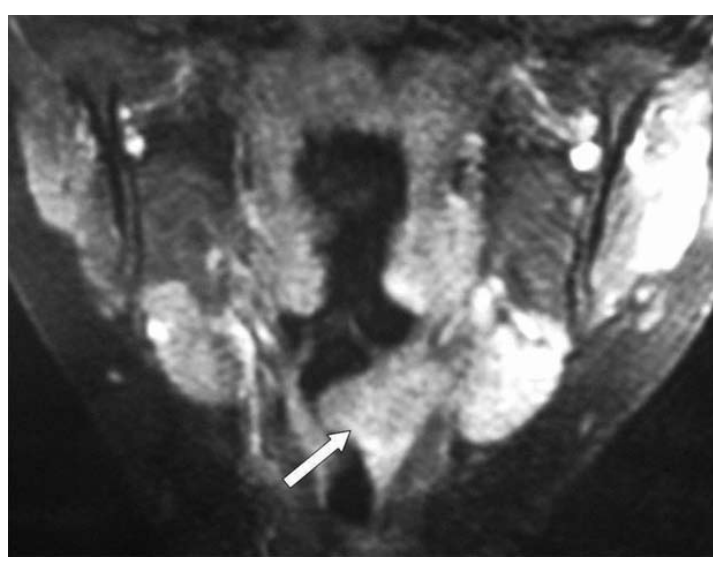

quetarlos como gigantes por no existir unos parámetros o criterios aceptados para hacerlo. La gravedad del cuadro clínico causado por el tumor nunca alcanzó los grados que su tamaño hacía prever, y en algunos casos se quedó muy por debajo de lo que se pensaba.

\section{Pacientes y métodos}

Son nueve pacientes (cinco mujeres y cuatro varones) con NF1 y NFP, localizados en el cuello, faringe, laringe y región torácica superior, identificados durante los primeros años de vida, la mayoría de ellos a través de estudios clínicos y de neuroimagen a lo largo de los últimos 20 años (1994-2013), lo que nos ha permitido determinar algunos signos específicos de los NFP espinales y paraespinales en pacientes con NF1. Cinco pacientes (casos 1, 2, 4, 5 y 8 ) habían heredado la NF1 y cuatro (casos 3, 6, 7 y 9) eran atribuibles a mutaciones de novo. Además, el estudio de este material permitía identificar otros tipos de neurofibromas en las regiones del cuello y torácica superior. Las edades de los pacientes cuando se hizo el diagnóstico estaban entre 3 y 15 años (edad media: 8,7 años), pero el estudio de algunos pacientes se inició algún tiempo antes, como en los casos 1, 3 y 9, cuando los tumores en el cuello no se sospechaban. Todos los pacientes vinieron enviados para estudio por los signos externos de NF1, principalmente manchas café con leche y otros sig- nos típicos. Siete pacientes mostraban una prominencia local en el cuello y uno presentaba prominencia en un lado de la orofaringe. Sólo un paciente (caso 3) desarrollaba estridor, dificultades respiratorias y problemas para dormir a los 12 años. Ninguno de los otros pacientes mostraba signos de obstrucción de las vías respiratorias o problemas durante el sueño.

Una paciente (caso 4) tenía un NFP occipital externo que infiltraba la fosa posterior y el cuello. Se hizo un intento quirúrgico para extirpar el tumor a la edad de 4 años en su provincia, pero se seccionó la arteria vertebral durante la intervención y el paciente fue remitido a nuestro servicio tras la ligadura de la arteria.

Todos los pacientes fueron sometidos a estudio de resonancia magnética (RM) de cabeza, cuello y mediastino, con planos coronal, sagital y axial. Estas imágenes eran los principales tests diagnósticos y los estudios de RM se repetían usualmente cada año en los cinco pacientes ( $\operatorname{casos} 1,3,4,5$ y 8 ) que fueron seguidos en nuestro servicio. Los pacientes 2, 6 y 9 se perdieron de vista; el caso 7 se consideró curado cinco años después de la exéresis completa del NFP, y el estudio de RM parecía confirmarlo. Los hechos clínicos y de imagen se muestran en la tabla.

\section{Resultados}

Algo más de la mitad (56\%) de los pacientes del grupo de estudio mostraba una historia familiar positiva de NF1. A pesar del considerable tamaño de los NFP, especialmente de los NFPVC, había pocos signos y síntomas clínicos relacionados con la afectación de la faringe, laringe y región torácica superior, o estaban ausentes. La laringe es, posiblemente, la localización menos frecuente de los NFPVC. Sólo un paciente (caso 3) tenía crecimiento intralaríngeo del tumor, que causaba problemas respiratorios con ronquidos durante el sueño desde los 6 años y, finalmente, obstrucción aérea. El tumor se le extirpó a los 12 años (Fig. 1), pero recidivó y a los 17 años necesitó una nueva extirpación. El NFP en los otros ocho pacientes era más lateralizado y mostraba un comportamiento más expansivo, desplazando muy lentamente las estructuras adyacentes sin causar problemas funcionales. El tumor pasaba al espacio intracraneal a través de la base craneal en tres pacientes (casos 2, 4 y 5) sin infiltrar el cerebro o el cerebelo, pero desplazando otras estructuras intracraneales (Fig. 2). El estudio histológico del tumor demostraba corresponder a NFP. 
Tabla. Hallazgos clínicos y de neuroimagen.

\begin{tabular}{|c|c|c|c|c|c|c|}
\hline & Sexo & $\begin{array}{l}\text { Localización } \\
\text { del NFP }\end{array}$ & $\begin{array}{l}\text { Edad al diagnóstico } \\
\text { y hallazgos clínicos }\end{array}$ & $\begin{array}{l}\text { Hallazgos en } \\
\text { resonancia magnética }\end{array}$ & Tratamiento & Evolución \\
\hline Paciente 1 & $\mathrm{~F}$ & $\begin{array}{l}\text { Cuello: lado D } \\
\text { Muy extenso }\end{array}$ & $\begin{array}{l}7 \text { años } \\
\text { NF1 cutánea } \\
\text { Prominencia SC en el lado D } \\
\text { Horner en el lado D } \\
\text { desde los } 7 \text { años }\end{array}$ & $\begin{array}{l}\text { NFP bilateral } \\
\text { Difuso y voluminoso, } \\
\text { extendido por el cuello, } \\
\text { faringe, laringe y mediastino, } \\
\text { más volumen en el lado D } \\
\text { Desviación de todas las estructuras } \\
\text { del cuello hacia el lado I }\end{array}$ & No & $\begin{array}{l}\text { Progresión hasta los } 11 \text { años; } \\
\text { después, sin cambios } \\
\text { A los } 20 \text { años, sin progresión del tumor } \\
\text { TDAH } \\
\text { Estudios medios-superiores (magisterio) }\end{array}$ \\
\hline Paciente 2 & $M$ & Cuello: Iado I & $\begin{array}{l}15 \text { años } \\
\text { NF1 cutánea } \\
\text { Prominencia SC en el lado I }\end{array}$ & $\begin{array}{l}\text { NFP entre la base } \\
\text { craneal y C6 en el lado I } \\
\text { Quiste aracnoideo } \\
\text { en la fosa temporal I } \\
\text { Tumor NOI }\end{array}$ & $\begin{array}{l}\text { Biopsia a los } 15 \text { años } \\
\text { Confirmación de NFP }\end{array}$ & $\begin{array}{l}\text { A los } 19 \text { años, tamaño del tumor estable } \\
\text { Estudia formación profesional }\end{array}$ \\
\hline Paciente 4 & $\mathrm{~F}$ & Cuello: Iado I & $\begin{array}{l}3 \text { años } \\
\text { Prominencia cutánea } \\
\text { y SC en el lado I } \\
\text { Dos neurofibromas en el } \\
\text { muslo I en la adolescencia }\end{array}$ & $\begin{array}{l}\text { NFP extracraneal occipital } \\
\text { NFP voluminoso } \\
\text { entre } \mathrm{C} 1 \text { y C4 en el lado I }\end{array}$ & $\begin{array}{l}\text { Extirpación parcial del } \\
\text { NFP del cuello a los } 4 \text { años }\end{array}$ & Acabó magisterio \\
\hline Paciente 5 & $\mathrm{~F}$ & $\begin{array}{l}\text { Cuello, faringe, } \\
\text { laringe y } \\
\text { mediastino } \\
\text { bilateral } \\
\text { Más volumen } \\
\text { en el lado D }\end{array}$ & $\begin{array}{l}4 \text { años } \\
\text { NF1 cutánea } \\
\text { Prominencia SC en el lado D } \\
\text { Horner en el lado D }\end{array}$ & $\begin{array}{l}\text { NFP difuso y voluminoso } \\
\text { entre la base craneal y la } \\
\text { zona alta del mediastino (D5) }\end{array}$ & No & $\begin{array}{l}\text { Progresión del NFP hasta los 11-12 años } \\
\text { A los } 19 \text { años, sigue en control } \\
\text { Asintomática } \\
\text { Nivel mental normal }\end{array}$ \\
\hline Paciente 8 & $\mathrm{~F}$ & $\begin{array}{l}\text { Cuello y } \\
\text { mediastino: } \\
\text { lado I }\end{array}$ & $\begin{array}{l}9 \text { años } \\
\text { NF1 cutánea } \\
\text { Prominencia en } \\
\text { el cuello: lado I } \\
\text { Horner en el lado I }\end{array}$ & $\begin{array}{l}\text { NFP muy voluminoso que se } \\
\text { extiende entre C1 y D5 del lado I } \\
\text { NFP menos voluminoso } \\
\text { en el lado D }\end{array}$ & No & $\begin{array}{l}\text { A los } 22 \text { años, vida normal } \\
\text { Sin crecimiento tumoral } \\
\text { desde los } 11 \text { años } \\
\text { Asintomática } \\
\text { Estudios de formación profesional } \\
\text { (cocina y pastelería) }\end{array}$ \\
\hline Paciente 9 & $\mathrm{~F}$ & $\begin{array}{l}\text { Cuello: } \\
\text { ambos lados }\end{array}$ & $\begin{array}{l}5 \text { años } \\
\text { NF1 cutánea } \\
\text { NFP cutáneo y } \\
\text { SC en ambos lados }\end{array}$ & $\begin{array}{l}\text { NFP entre C1 y C3 } \\
\text { de ambos lados }\end{array}$ & No & $\begin{array}{l}\text { A los } 17 \text { años, asintomática } \\
\text { Nivel mental normal }\end{array}$ \\
\hline
\end{tabular}

D: derecho; F: femenino; I: izquierdo; M: masculino; NF1: neurofibromatosis tipo 1; NFP: neurofibroma plexiforme; NOI: secuencia nucleotídica de interés; SC: subcutáneo; TDAH: trastorno por déficit de atención/hiperactividad. 
Figura 2. Caso 4: niña de 3 años. a) Estudio coronal de resonancia magnética enriquecida con gadolinio que muestra el neurofibroma plexiforme localizado en la región cervical superior que cruza la base craneal en la zona del foramen occipital; el tumor comprime la zona inferior del hemisferio cerebeloso izquierdo y desvía la vallécula de Magendie hacia el lado derecho (flecha); b) La vista axial del mismo estudio muestra la parte extracraneal del neurofibroma en la región posterior de la cabeza y la extensión intracraneal del tumor en el lado izquierdo del cuello, con desplazamiento de la faringe hacia el lado derecho (flecha).
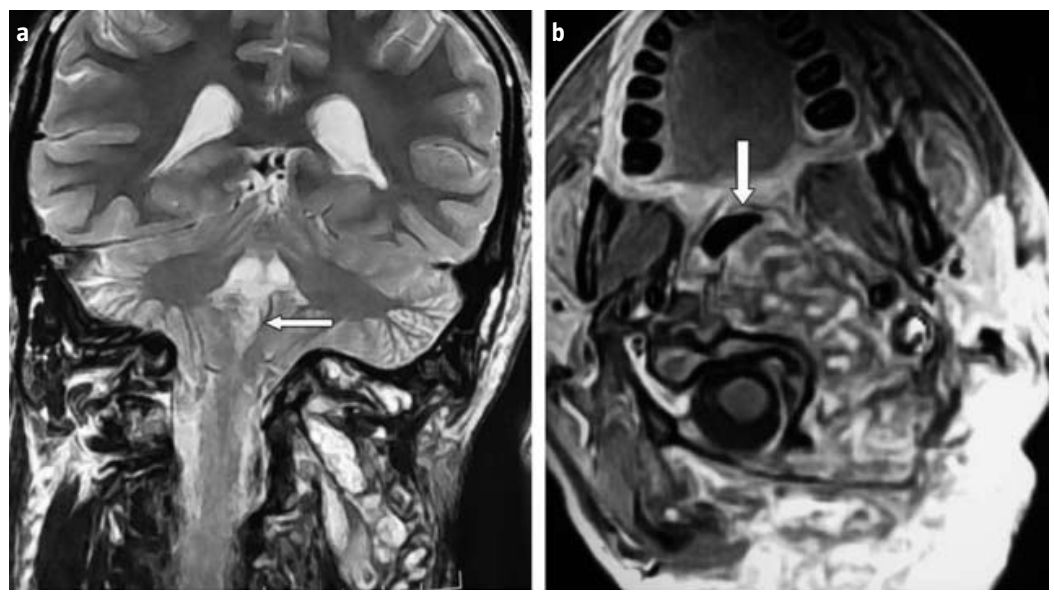

Figura 3. Caso 8: cortes coronal (a) y sagital (b) del estudio de resonancia magnética con gadolinio de una niña de 10 años con neurofibromatosis tipo 1, clínicamente asintomática, que no aceptó someterse a más estudios de imagen, tratamiento ni revisiones clínicas periódicas ni de ningún otro tipo. Sólo se comunica anualmente con nosotros por teléfono. Actualmente, con 22 años, se encuentra asintomática.
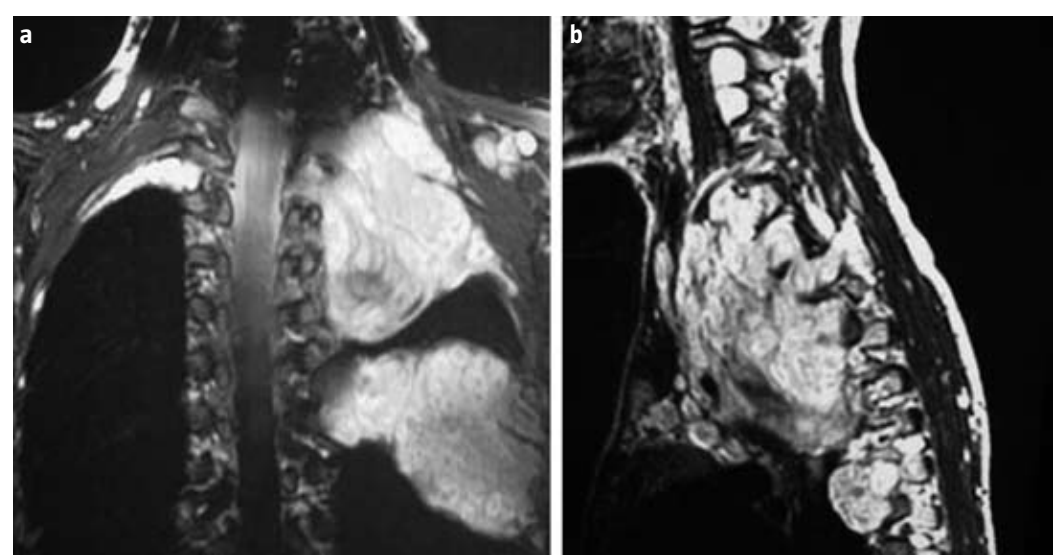

Tres pacientes (casos 1,5 y 8 ) mostraban significativa desviación de la faringe, laringe, esófago o tráquea, causada por el NFP; a pesar de la presencia bilateral, el NFP mostraba importantes diferencias del tamaño tumoral entre ambos lados del cuello.
La RM demostraba desviación de todas las estructuras del cuello, incluyendo vasos (arterias carótidas y venas yugulares), faringe, laringe, tráquea y esófago. El grado de desplazamiento dependía del volumen del tumor y éste, a su vez, estaba relacionado con el tiempo de evolución, pero sólo hasta la preadolescencia, es decir, hasta los 11-12 años. La gravedad de los síntomas, sin embargo, no estaba proporcionada con el tamaño del tumor ni con la marcada desviación de todos los órganos y vasos del cuello y parte superior del tórax en estos casos. El diagnóstico de NFP es histológico, pero la confirmación de este diagnóstico está casi siempre en las imágenes de NFP, que aparecen en el estudio de RM con origen tumoral en varias raíces medulares en la mayoría de los casos, si bien se podían apreciar varios abultamientos semiindependientes del tumor en algunos pacientes, hecho muy evidente en el caso 8 (Fig. 3), que llegó a alcanzar un tamaño muy voluminoso en algunas partes, como la dorsal y la zona de transición dorsocervical. La paciente había sido estudiada a los 9 años en otro hospital no por mostrar síntomas clínicos, sino por manchas café con leche. En el hospital de procedencia le practicaron estudios de RM y, en vista de los hallazgos, semejantes a los de la figura, nos remitieron a la paciente, que, un año más tarde, mostró las imágenes presentadas en el trabajo, pero con resultados exploratorios, por otra parte, normales. Ante la prescripción terapéutica de extirpación de los NFP, abandonó el hospital y volvió a su casa, negándose a cualquier tratamiento y a estudios posteriores. Accedió sólo a comunicarse por teléfono personalmente con nosotros siempre que la llamamos (vive en una provincia fuera de Madrid), cosa que hacemos cada año o cuando necesitamos alguna información. Los dos voluminosos NFP de la cavidad torácica izquierda y otros de menor tamaño en la zona izquierda del cuello parece que no le han causado ningún problema, su actividad física es normal (no hace deporte), y su rendimiento escolar ha sido normal, finalizando estudios medios y formación profesional en cocina y pastelería, ámbito en el que trabaja normalmente. No se ha prestado a practicarse nuevos estudios de RM, por lo que ignoramos la evolución del tamaño de los NFP del tórax y del cuello. No precisa dormir en posición incorporada ni muestra más fatiga que otras personas con el ejercicio físico.

Las otras dos pacientes (casos 1 y 5 ) han seguido control con nosotros desde los 4,5 años hasta la actualidad, que cuentan con 20 y 19 años, respectivamente. El estudio de RM (Fig. 4) practicado cada año ha permitido conocer la evolución volumétrica de estos tumores y de cualquier otro de la región. 
Se observó un progresivo incremento del tamaño tumoral durante los estudios anuales de RM hasta que la voluminosa masa tumoral ocupaba el espacio prevertebral y desplazaba bilateralmente todas las estructuras internas del cuello y de la región torácica superior, desde el lado donde el tumor era más voluminoso hasta el otro lado del cuello, erosionando también los cuerpos de las vértebras cervicales (Fig. 4) en las tres mujeres, que nunca presentaron problemas respiratorios, vasculares, del sueño, para tragar o de tipo doloroso. Las arterias vertebrales no eran desplazadas por el tumor, pero mostraban importante asimetría, y eran más delgadas en el lado donde el NFP era más voluminoso. El tumor crecía y alcanzaba gran tamaño hasta los 11-12 años en las dos pacientes cuya evolución radiológica seguimos, hasta alcanzar un tamaño voluminoso en ambas (casos 1 y 5), y los estudios de RM mostraban ausencia del incremento del tamaño tumoral a partir de dicha edad (probablemente el caso 8, en el que no hay control radiológico después de los 10 años), pero tampoco disminución, y las tres pacientes, que actualmente cuentan con 20, 19 y 22 años, respectivamente, están aparentemente normales desde el punto de vista clínico y realizando estudios secundarios con rendimiento promedio. Probablemente, el mayor incremento del tumor tiene lugar durante los primeros años de vida.

El NFP se identificó tras la RM practicada porque los pacientes tenían NF1. El neurofibroma externo se abordó quirúrgicamente, pero se seccionó la arteria vertebral izquierda durante la cirugía, lo que requirió ligadura de esta arteria y quedó una leve hemiparesia derecha. La extirpación completa del NFP se llevó a cabo en un paciente (caso 7), y la extirpación parcial, en dos (casos 3 y 4). En el caso 2 sólo se obtuvo una biopsia del tumor. Se utilizó la imagen preoperatoria y postoperatoria para determinar la parte resecada del tumor en los tres pacientes. Los tres pacientes con los tumores voluminosos del cuello (casos 1, 5 y 8) no aceptaron la extirpación del tumor, que en ninguno de ellos podría haber sido total.

\section{Discusión}

Los NFP son tumores típicamente congénitos, la mayoría de las veces benignos. La historia natural de su patrón de crecimiento no se describe bien habitualmente. El NFP se extiende frecuentemente por largos segmentos de algunos nervios. El neoplasma intrafascicular puede extenderse centrípetamente a
Figura 4. Caso 5: estudios de resonancia magnética en una niña con neurofibroma plexiforme voluminoso en el cuello (NFPVC) a diferentes edades. Imágenes de resonancia magnética en proyección axial enriquecida con gadolinio a los 4,5 (a), 5,5 (b), 8 (c) y 16 años (d). A esta edad (d) se aprecia imagen del NFPVC en localización prevertebral, con desgaste de las vértebras cervicales y deformación de toda la columna cervical, que muestra inversión de su curvatura.
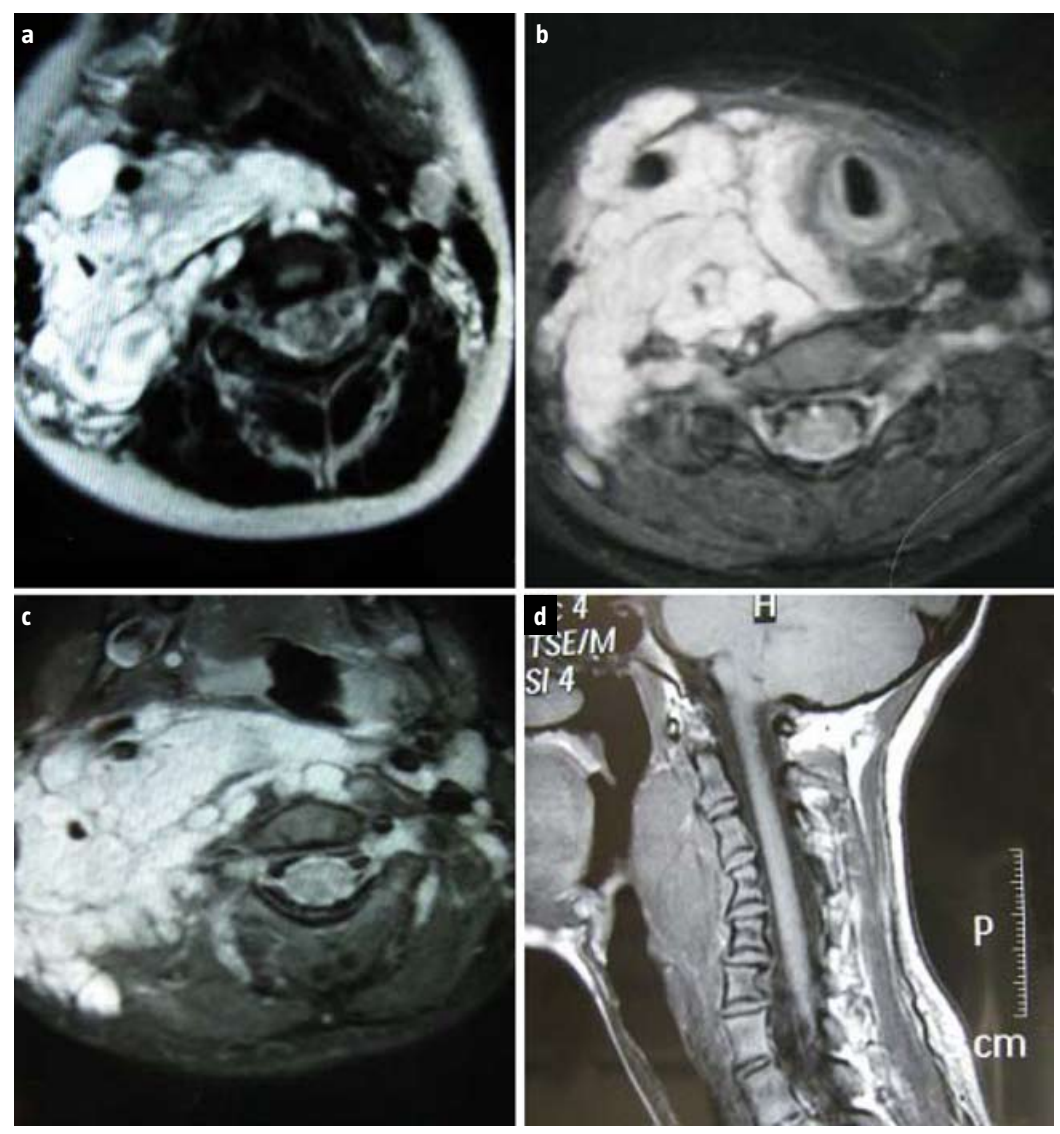

lo largo del nervio hasta la médula espinal, y parece crecer en la primera niñez a ritmo variable con fases de crecimiento y de detenciones [5]. Estos tumores se comportan como lesiones difusas, pobremente definidas, que tienen crecimiento lento, pero que pueden ser localmente agresivas. Tienden a infiltrar los tejidos de las áreas adyacentes; en un principio, de las zonas más periféricas cuyos tejidos vecinos ofrecen menos resistencia, y en la última fase, también lo hacen de las zonas prevertebrales. La extirpación completa de los NFP es extremadamente difícil.

Todos los NFP del cuello de nuestra serie parecían radiológicamente estar derivados de raíces medulares cervicales de uno o de ambos lados, y también de las primeras raíces dorsales en el caso 8 . 
En otras series, estos tumores provenían del nervio vago [5]. Los NFP del cuello se han referido a menudo como neurofibromas laríngeos. Posiblemente, Suchanek publicó el primer caso de neurofibroma laríngeo en 1925 [13]. Una revisión de la bibliografía refería 35 casos de todas las edades en 1987 [14]. Aunque el 25-35\% de NFP en casos con NF1 ocurren en la cabeza y el cuello, la localización en la laringe es rara [15].

Sólo un paciente (caso 3) de nuestra serie presentaba localización laríngea del NFP sobre la cuerda vocal izquierda. En una serie de 74 pacientes con NFP en cualquier región del cuerpo, sólo tres estaban localizados en la lengua y la orofaringe [16]. La localización más común de los NFPVC del cuello es la región interna de un lado (menos frecuentemente de ambos lados) del cuello y se produce el desplazamiento de las estructuras contenidas en la región (arterias, venas, faringe, laringe y esófago) por la masa tumoral. Se han publicado muy pocos casos pediátricos de NFP retrofaríngeos [12]. La compresión de la médula cervical por neurofibromas originados en las raíces de la zona constituye un problema clínico importante en pacientes con NF1, pero estos tumores se refieren raras veces [3,8]. Más que las imágenes neurorradiológicas, los déficits neurológicos progresivos deberían ser los que dictaran la necesidad de la cirugía [8]. La mayoría de los casos publicados durante las últimas dos décadas ha ocurrido en niños [4,6-8,10-12,16-18], incluyendo la presentación en un recién nacido con apnea causada por el tumor localizado en la unión craneocervical con compresión de la zona cervical superior y la bulbar inferior, que causaba apnea completa y, finalmente, la muerte a la edad de 5 meses [3]. La mayoría de los tumores es asintomática y, en nuestra serie, sólo un paciente (caso 3) tenía crecimiento del tumor intralaríngeamente y causaba obstrucción del paso del aire, además del ensanchamiento del cuello en el lado con el NFP laríngeo, que requería resección y repetición de la intervención quirúrgica cinco años después por recidiva. Hasta la fecha, no hay referencias de una publicación de extirpación completamente exitosa de un NFP laríngeo, incluso después de una laringuectomía total [15]. La recurrencia y la resección posterior son la regla. En nuestro grupo de estudio, sólo discretos ensanchamientos y abultamientos no dolorosos hicieron sospechar la presencia de un tumor sin especificación de su tamaño, incluso en los tres pacientes con masivo NFP ( $\operatorname{casos} 1,5$ y 8). El tumor mantenía mayor crecimiento durante los primeros años de vida, antes de los 5 años, seguido del período comprendido entre 5-11 años. A esta edad había cese del crecimiento del tumor, que era sugestivo de que se había alcanzado una prolongada fase de paralización de la evolución tumoral. Tras 5-9 años de la detención del crecimiento de estos tres voluminosos NFP, que son los casos cuya evolución hemos seguido más tiempo, éstos siguen manteniendo el mismo tamaño, y los tres pacientes, todos mujeres, continúan estando asintomáticos a pesar del enorme tamaño tumoral y el marcado desplazamiento de las estructuras anatómicas del cuello. El signo de Horner aparecía en el lado ipsilateral del tumor a los 4-7 años en estos tres pacientes, lo que significaba que el tumor, en su crecimiento, había alcanzado el simpático cervical, y en el caso 7, después de extirpar el tumor del cuello.

La edad, la extensión de la resección y la localización del NFP son variables independientes que afectan al resultado de la cirugía de estos tumores [4]. La resección completa del tumor sólo es posible en pacientes con NFP pequeños [9]. La extirpación de menos del $90 \%$ de la masa tumoral conduce a recurrencia en al menos el 60\%, mientras que la resección total o subtotal del tumor asegura porcentajes de recurrencia inferiores al $20 \%$ y $40 \%$, respectivamente [4]. Pacientes con edades inferiores a los 10 años tienen gran riesgo de recurrencia [4]. Debido a ello, algunos autores creen que es prudente retrasar la cirugía tanto como sea posible en aquellos pacientes pediátricos asintomáticos con NFP del cuello y de la cara [10]. Sin embargo, solamente la cirugía mejora los síntomas causados por la obstrucción aérea [9].

Se ha realizado faringotomía lateral con hemilaringuectomía supraglótica en algunos casos para mejorar los síntomas obstructivos y obtener una biopsia del tumor [11].

\section{Bibliografía}

1. Conference NIHCD. Neurofibromatosis: conference statement. Arch Neurol 1988; 45: 575

2. Viskochil D, Buchberg AM, Xu G, Cawthon RM, Stevens J, Wolff RK, et al. Deletions and a translocations interrupt a cloned gene at the neurofibromatosis type 1 locus. Cell 1990; 62: 187-92.

3. Clarke DB, Farmer JP, Montes J, Guatters GV, Rouleau G. Newborn apnea caused by a neurofibroma at the craniocervical junction. Can J Neurol Sci 1994; 21: 64-6.

4. Needle MN, Cnaan A, Datilo J, Chatten J, Phillips PC, Chochat S, et al. Prognostic signs in the surgical management of plexiform neurofibroma: the Children's Hospital of Philadelphia experience. J Pediatr 1997; 131: 678-82.

5. Wise JB, Cryer JE, Belasco JB, Jacobo I, Elden L. Management of head and neck plexiform neurofibromas in pediatric patients with neurofibromatosis type 1 . Arch Otolaryngol Head Neck Surg 2005; 131: 712-8.

6. Martin DS, Stith J, Awwad EE, Handler S. MR in neurofibromatosis of the larynx. AJNR Am J Neuroradiol 1995; 16: 503-6. 
7. Ranson ER, Yoon C, Manolidis S. Single stage near total resection of massive pediatric head and neck plexiform neurofibromas. Int J Pediatr Otorhinolaryngol 2006; 70: 1055-61.

8. Leonard JR, Ferner RE, Thomas N Gutman DH. Cervical cord compression from plexiform neurofibromas in neurofibromatosis 1. J Neurol Neurosurg Psychiatry 2007, 78: 1404-6.

9. Serletis D, Parkin P, Bouffet E, Shroff M, Drake JM, Rutwa JT. Massive plexiform neurofibromas in childhood: natural history and management issues. J Neurosurg 2007; 106 (Suppl Pediatrics): S363-7.

10. Wise JB, Patel SG, Shah JP. Management issues in massive pediatric facial plexiform neurofibroma with neurofibromatosis type 1 . Head Neck 2002; 24: 207-11.

11. Yucel EA, Guldiken Y, Ozdemir M, Ozturl AS. Plexiform neurofibroma of the larynx in a child. J Laryngol Otol 2002; 116: 49-51.

12. Piazza ES, Donnellan KA, Goodier AM, Carron JD. Massive retropharyngeal neurofibroma in a pediatric patient. Int J Pediatr Otorhinolaryngol 2010; 74: 939-41.

13. Suchanek E. Neurinom des Kehlkopfeinganges. Monotsschr Ohrenheilkd Laryngo-rhinol 1925; 50: 613-7.

14. Garabedian EN, Riviere F, Cotin G, Ménard M. Localisations laryngés de la maladie de Recklinghausen. Ann Pediatr (Paris) 1987; 34: 443-6.

15. Dave SP, Farooq U, Civantos FJ. Management of advanced laryngeal and hypopharyngeal plexiform neurofibromas in adults. Am J Otolaryngol 2008; 29: 279-83.

16. Waggoner DJ, Towbing J, Gottesman G, Gutmann DH. Clinical-based study of plexiform neurofibromas in neurofibromatosis 1. Am J Med Genet 2000; 92: 132-5.

17. Manolidis S, Higuera S, Boyd V, Hollier LS. Single-stage total and near-total resection of massive pediatric head and neck neurofibromas. J Craniofac Surg 2006; 17: 506-10.

18. Masip MJ, Esteban E, Alberto C, Menor F, Cortina H. Laryngeal involvement in pediatric neurofibromatosis: a case report and review of the literature. Pediatr Radiol 1996; 26: 488-92.

\section{Voluminous plexiform neurofibromas of the neck region in neurofibromatosis 1}

Aim. To present the clinic, imaging and evolutive characteristics of a series of patients with neurofibromatosis 1 with voluminous plexiform neurofibromas in the neck (VPNFN) during childhood.

Patients and methods. Nine patients (five females and four males) who were diagnosed as VPNFN at ages between 3 and 15 years. The VPNFN widespread to the posterior fossa or the upper thoracic region in some cases. The diagnosis was based on the clinical, imaging and histological findings.

Results. One of the tumors was intralaryngeal and caused respiratory difficulties. The other eight patients had the origin of the tumor in several spinal roots of one or both sides and could growth to the posterior fossa and to the upper thoracic region in some cases with displacement of the surrounding organs, especially in three patients, all girls, in whom the tumor reached a voluminous size on one side, that was observed only until 10 to 11 years when the growth ceased.

Conclusions. The VPNFN are histologically benign tumors. Those located in the larynx must be removed because of the respiratory problems, but it is not necessary in cases with other locations despite the voluminous size that can reach in some patients with great displacement of the surrounding organs. The analysis of the results of our series may demonstrate that al least the extralaryngeal tumors only grow to 11-12 years of age. This possibility may make recommendable to retard the surgical treatment as much as possible in cases that it is not necessary.

Key words. Mediastinum plexiform neurofibroma. Neurocutaneous disorders. Neurofibromatosis 1. Pharynx plexiform neurofibroma. Voluminous plexiform neurofibromas in the neck. 